Muséologies

Les cahiers d'études supérieures

muséologies

\title{
De l'observation à l'appropriation : une démarche proposée pour le (jeune) visiteur dans le projet « Galerie des Enfants " au Muséum
}

\author{
Didier Julien-Laferrière, Agnès Parent et Isabelle Legens
}

Volume 3, numéro 2, printemps 2009

URI : https://id.erudit.org/iderudit/1033574ar

DOI : https://doi.org/10.7202/1033574ar

Aller au sommaire du numéro

Éditeur(s)

Association Québécoise de Promotion des Recherches Étudiantes en Muséologie (AQPREM)

ISSN

1718-5181 (imprimé)

1929-7815 (numérique)

Découvrir la revue

Citer cet article

Julien-Laferrière, D., Parent, A. \& Legens, I. (2009). De l'observation à

l'appropriation : une démarche proposée pour le (jeune) visiteur dans le projet

"Galerie des Enfants " au Muséum. Muséologies, 3(2), 202-213.

https://doi.org/10.7202/1033574ar 
Article quinze

\section{De l'observation à l'appropriation:}

\section{une démarche proposée pour le (jeune) visiteur \\ dans le projet «Galerie des Enfants » au Muséum}

APRĖS UNE FORMATION UNIVERSITAIRE SCIENTIFIQUE, DIDIER JULLIEN-LAFERRIĖRE A INTÉGRÉ LE MUSÉUM COMME CONCEPTEUR DEXPOSITION EN 1994. IL EST INIERVENU SUR DIVERS PROJETS DEXPOSITIONS TEMPORAIRES ET DE RÉNOVATION DELEXPOSITION PERMANENTE DE LA GRANDE GALERIE DE LEVVOLUTION. AGNĖS PARENT A UNE FORMATION UNIVERSITAIRE EN COMMUNICATION SCIENTIFIQUE. APRÈS UNE ACTIVITÉ D'ÉVALUATION DEXPOSITIONS, ELLE A REJOINT LE MUSÉUM EN 1998 COMME RESPONSABLE MULTIMÉDIA, FONCTION QUELLLE ASSURE SUR LES EXPOSITIONS TEMPORAIRES DE LA GRANDE GALERIE DE LEVVOLUTION ET SUR LES GALERIES DU JARDIN DES PLANTES. ELLE EST ÉGALEMENT CONCEPTRICE DEXXPOSITION ET CHEF DE PROJET DE LA GALERIE DES ENFANTS. ISABELLELEGENS, APRĖS UNE FORMATION LITTERAIRE SUIVIE DUN TROISIÈME CYCLE EN MULTIMÉDIA EST CONCEPTRICE MULTIMÉDIA INDÉPENDANTE SPÉCIALISÉE DANS LA CONCEPTION DE DISPOSITIFS LUDO-ÉDUCATIFS, ELLE COLLABORE RÉGULIËREMENT AUX EXPOSITIONS TEMPORAIRES DU MUSÉUM DEPUIS 2003. [julien@mnhn.fr] 


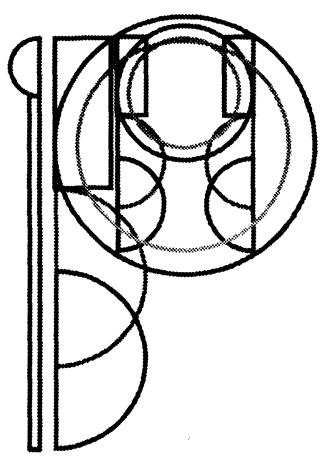

Pourquoi un nouvel espace d'exposition pour les enfants?

Depuis 1994, année d'ouverture de la Grande Galerie de l'Évolution, la proportion du public familial, c'est-à-dire les enfants accompagnés, est en progression constante. Inversement, l'offre qui leur est proposée reste limitée. La « salle de découverte» qui leur est destinée est de surface réduite, peu intégrée au parcours général et peu scénographiée. Grâce aux animaux naturalisés spectaculaires, l'exposition permanente est attractive pour les plus petits, tandis que les plus grands apprécient la richesse du contenu des balcons, mais elle est peu adaptée aux enfants d'âge intermédiaire. Par ailleurs, les attentes et les préoccupations des visiteurs, notamment sur les enjeux environnementaux, ont évolué et ont pris une grande importance depuis l'ouverture de la Grande Galerie de l'Évolution.

C'est pourquoi le projet de "Galerie des Enfants " a été lancé, plus particulièrement à destination des enfants de 6 à 12 ans, et centré autour du mot clé de "biodiversité». Il porte sur l'ensemble de l'espace pédagogique et culturel actuel, soit la salle de découverte, mais aussi sur les quatre espaces d'activités et les locaux techniques associés, sur 550 mètres carrés au sol et 300 mètres carrés de mezzanine. 
Les grandes orientations du projet et comment elles ont été définies

À partir d'une phase d'orientation préliminaire, nous avons proposé un espace pour les enfants accompagnés, en famille mais aussi en groupe scolaire ou de loisir, sans oublier les enfants porteurs de tout type de handicap. L'analyse en interne des besoins ainsi que l'intervention d'une équipe de programmation nous ont conduits à définir une réorganisation complète de l'espace. Le programme consiste en effet à démonter la mezzanine actuelle, à reconstruire deux salles d'activités superposées et à réserver la plus grande partie de la superficie (soit plus de 300 mètres carrés au sol) à un espace d'exposition sur deux niveaux grâce à la construction d'une plateforme intégrée au projet scénographique.

Pendant la phase d'orientation, nous avons visité de nombreux sites muséaux, en France, en Europe et en Amérique du Nord, et rencontré de nombreux professionnels de l'enfance et du public enfant des musées. Au cours de nos visites, nous avons ainsi approché des types très divers d'expositions pour les enfants: parcours enfants intégré, exposition-atelier encadrée par des animateurs, " musée des enfants " à l'anglo-saxonne, en visite libre ou dirigée, exposition de type centre de ressources, exposition multi-support. Nos échanges avec les enseignants, les didacticiens et les psychologues nous ont appris, ou confirmé, qu'il existait une réelle rupture autour de sept ans concernant, outre l'apprentissage de la lecture, l'accès à des concepts abstraits et la découverte par l'enfant du monde qui l'entoure et que, par ailleurs, les enfants de cet âge étaient non seulement curieux de nature, mais aussi connaisseurs. Ce sont deux éléments clés qui nous ont guidés tout au long de l'élaboration du programme muséologique. Pendant cette phase, un comité de pilotage, constitué d'un groupe de personnalités d'horizons divers, a été constitué. Ce comité est consulté et a un rôle de référence à chacune des grandes étapes du projet.

Nous avons dû intégrer au projet les paramètres liés au contexte du lieu et de l'institution: un espace inclus dans un espace historique à l'identité forte, la Grande Galerie de l'Évolution; un outil de médiation privilégié, la collection scientifique; l'absence de spécimens vivants, pour des raisons essentiellement techniques (et bien que l'on connaisse l'attrait des enfants pour le vivant); un espace fonctionnant en libre-service, la présence d'animateurs devant rester optionnelle; et enfin un espace à aménager tout en longueur (11 mètres de large sur 50 de long) et à remettre à niveau du point de vue technique (traitement de l'air, traitement acoustique, normes de sécurité notamment). 


\section{Aperçu du parcours conceptuel}

L'exposition est structurée en deux grandes parties: le visiteur est tout d'abord invité à partir à la découverte de trois milieux, ou écosystèmes. Ces trois milieux sont clairement identifiés et leur présentation s'appuie sur la réalité, de façon à leur donner un caractère concret et incarné. La découverte de chaque milieu est conceptuellement structurée en deux temps. Dans un premier temps, le visiteur peut observer les plantes et les animaux peuplant ces milieux - sous forme de spécimens naturalisés pour les animaux, d'illustrations réalistes pour les plantes - et en apprendre un peu plus sur eux, leur mode de vie et leurs interactions, par l'intermédiaire de différents dispositifs. Dans un deuxième temps, d'autres dispositifs permettent d'élargir le propos au fonctionnement d'ensemble de l'écosystème ainsi qu'aux enjeux qui y sont liés : les aspects des impacts de l'Homme, de la gestion et de la conservation.

Le parcours commence par le milieu qui nous est généralement le plus proche, la ville, avec l'exemple de Paris, le lieu où l'on se trouve. Cette première entité se conclut par un jeu collectif qui invite à imaginer la ville de demain dans son aspect multifonctionnel. Le parcours se poursuit avec la rivière, un écosystème que l'on peut côtoyer par exemple pendant les promenades du dimanche, mais qui reste mystérieux. L'exemple choisi est celui de la Bassée, une portion de la vallée de la Seine en amont de Paris, dont la biodiversité est particulièrement riche. La partie rivière se termine par le jeu du " petit aménageur " qui, en jouant à l'ingénieur virtuel, aura pour mission de restaurer le peuplement de poissons et pourra ainsi voir les conséquences positives ou négatives de ses actions. Enfin, le visiteur est invité à prendre du recul en allant explorer un milieu éloigné de notre environnement familier, la forêt tropicale des Indiens Kayapo en Amazonie brésilienne. Dans ce lieu emblématique de la biodiversité, il fait connaissance entre autres avec le peuple indien qui vit en immersion dans la forêt et qui, par sa présence, a contribué à sa préservation.

La deuxième partie prolonge l'élargissement conceptuel puisqu'elle aborde l'échelle de la planète. Installée sur une plateforme surplombant les trois milieux particuliers précédemment traversés, elle permet d'appréhender la biodiversité à l'échelle du globe, de prendre conscience de quelques problématiques environnementales globales - en particulier le réchauffement climatique et l'appauvrissement de la biodiversité - et d'aborder comment, individuellement et collectivement, chacun peut contribuer à un meilleur état de la planète. 
JONCHERY, Anne. Quand la famille vient au musée: des pratiques de visites aux logiques culturelles. Thèse de doctorat. Muséum national d'histoire naturelle, 2005.

\section{Quel langage muséographique?}

L'exposition vise avant tout à éveiller la curiosité, à sensibiliser, à susciter la réflexion, dans un cadre d'éducation dite informelle. Sa muséographie s'appuie largement sur l'animal naturalisé, à la fois outil privilégié dans un musée d'histoire naturelle, vecteur d'émotion et support d'interrogation, individuelle et collective. L'espace d'exposition se veut un lieu d'interaction et d'échange au sein du groupe ou de la cellule familiale. On sait en effet que l'interaction entre visiteurs est un vecteur fort d'enrichissement à la visite. Une étude de public récente ${ }^{[1]}$ a d'ailleurs montré que le fait de partager une activité, de faire quelque chose ensemble, constituait l'un des principaux motifs de visite du musée en famille.

La question de l'organisation du parcours se posait également. Comment se positionner entre un espace totalement ouvert, où l'enfant aura tendance à papillonner d'un dispositif à un autre, et un parcours très guidé, où "l'écoulement» régulier des visiteurs est plus difficile à contrôler ? Sachant par ailleurs que l'espace devait être en mesure d'accueillir une classe entière, soit 20 à 30 jeunes en même temps, dans le cadre des visites scolaires, nous avons conçu l'exposition selon une trame narrative relativement lâche, correspondant à un parcours semi-dirigé. Les trois parties "milieux" et la partie "planète" s'enchaînent en quatre unités à l'intérieur desquelles le parcours est libre, chacune d'elles étant prévue pour occuper une classe pendant une quinzaine de minutes. Parallèlement, à l'intérieur de chaque espace et sur l'ensemble du parcours, nous avons cherché à ménager un rythme de visite en alternant des activités individuelles et collectives, des phases plus calmes d'observation et des phases plus actives, des dispositifs aux temps de consultation brefs ou plus longs, tout cela réparti de façon relativement homogène dans l'espace.

\section{Un aperçu scéno- et muséographique}

La scénographie, conçue par l'Atelier de scénographie Pascal Payeur, repose sur un traitement contrasté des quatre unités de l'exposition. Répondant à une demande de scénographie « immersive » du programme muséologique, le traitement des trois parties " milieux" repose sur une évocation, plutôt qu'une reconstitution, des milieux en question. La ville est dominée par des structures verticales anguleuses qui évoquent les immeubles et les bâtiments. Les cachettes, les recoins, les éléments dispersés (oiseaux, insectes, petites plantes) y sont nombreux, une manière de souligner le caractère discret et souvent inattendu de la présence animale et végétale dans le monde urbain. 
L'espace consacré à la rivière et aux milieux qui la bordent a au contraire une dimension horizontale. Spécimens et dispositifs sont portés par des plateaux en verre aux formes arrondies, évoquant la surface de l'eau et reflétant le plafond, lui-même animé par des jeux de lumière évoquant le ciel nuageux. Une partie du sol est également transparente, donnant au visiteur l'impression inattendue d'évoluer entre les rives, comme s'il était sur une barque.

La forêt tropicale est quant à elle dominée par les « troncs » d'arbres, structures en bois hautes et larges portant et abritant dispositifs et spécimens. Dans le fond, une grande photo rétro-éclairée, contrastant avec l'ambiance générale relativement sombre, évoque une clairière. La forêt se prolonge ensuite au début du niveau supérieur où l'on monte métaphoriquement dans la canopée, avant de déboucher dans la partie «planète».

La "planète», par contraste, est constituée d'une plateforme dégagée ménageant un large point de vue. Elle est comme posée sur les "milieux" qu'elle surplombe et avec lesquels elle entre en communication.

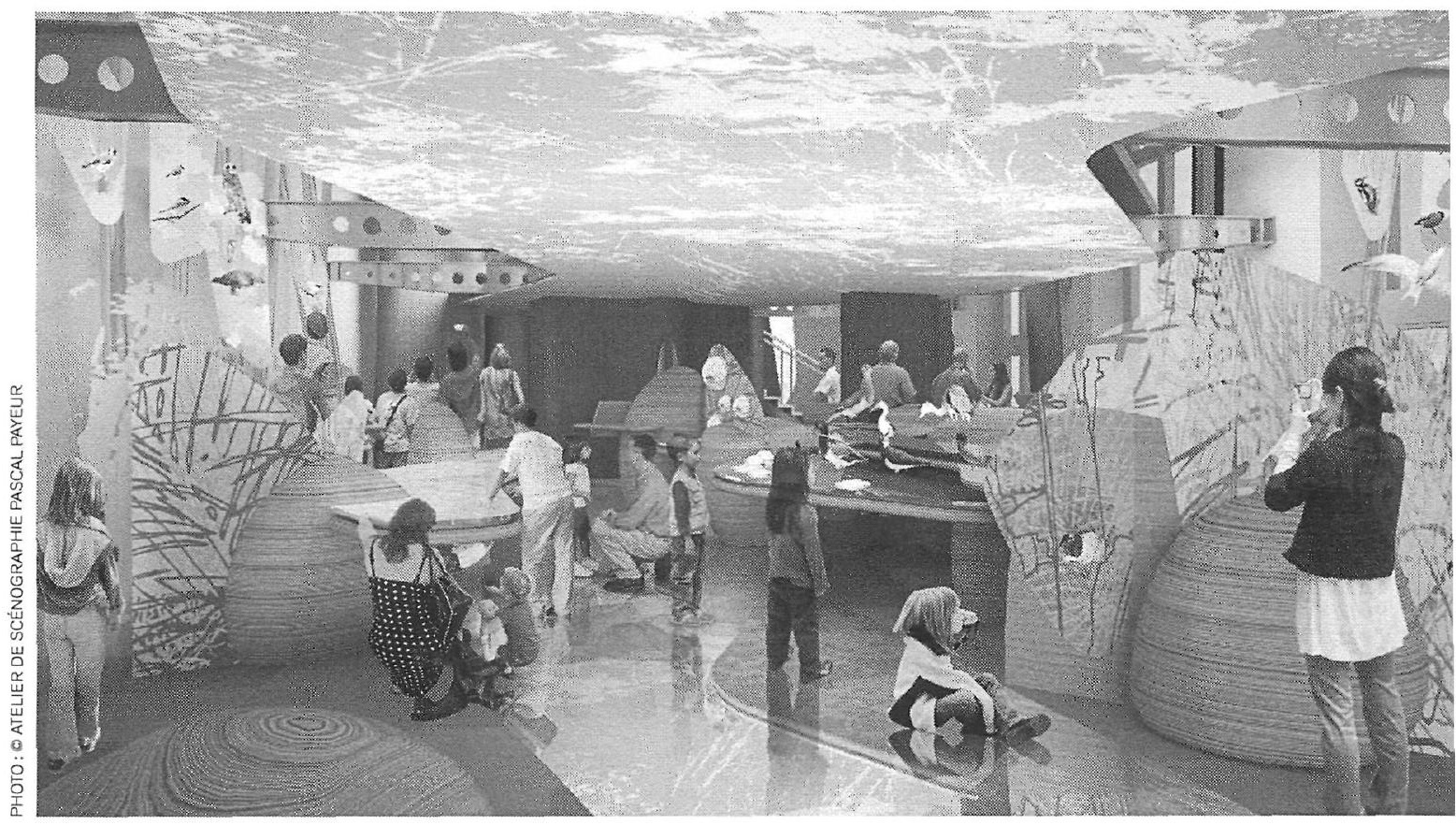

Vue de synthèse de la partie rivière, juillet 08 (image de travail). 
Quelques exemples de dispositifs:

- pour découvrir les oiseaux du jardin en ville : un jeu multimédia permet de reconnaître les chants de quelques oiseaux présentés sous forme de spécimens naturalisés;

- pour se rendre compte de la différence de texture de la peau des poissons : trois moulages à toucher à destination des personnes aveugles comme des autres (l'exposition est ainsi ponctuée d'une quinzaine d'objets à toucher, avec contenu adapté aux aveugles);

- des activités faisant appel au corps : pour aller à la rencontre de la souris, se glisser dans un trou où l'on se retrouve à son échelle (sur un écran);

- des «manips » simples: tourner une roue pour faire défiler les saisons de la forêt tropicale et les comparer avec les saisons de chez nous;

- des dispositifs jouant sur l'effet de surprise : mettre ses pas dans les empreintes du jaguar pour l'éclairer, alors qu'il est tapi derrière une vitre sans tain;

- pour interroger des spécimens ou des objets et ainsi chercher ce qu'ils recèlent comme information cachée : viser les oiseaux en manœuvrant une borne orientable, " attraper " les images des poissons à l'aide d'une table tactile, "faire parler " un objet usuel par l'intermédiaire d'un écran (où se cachent les poissons dans la rivière et comment font-ils pour tous vivre au même endroit? comment utiliser au mieux tel objet du quotidien pour diminuer notre impact sur la planète?);

- pour se plonger dans la vie nocturne de la ville: entrer dans un petit théâtre plongé dans l'obscurité où renard, chouette hulotte et autre faune nocturne sont mis en scène en mêlant spécimens naturalisés et vidéos;

- pour découvrir comment la ville change au fil du temps : un dispositif permet de se déguiser et de s'incruster dans un film présentant la vie à Paris à différentes époques, du temps des hommes préhistoriques à celui de nos grands-parents ;

- pour réfléchir ensemble aux solutions pour "sauver la planète» : une table des débats invite à donner son opinion et permet de connaître les avis des autres visiteurs sur les questions environnementales;

- et, pour finir sur une note plus légère : une réalisation collective par l'intermédiaire de tablettes graphiques où chacun peut exprimer en dessin sa vision de la nature. 
Le projet, en cours, en est actuellement à la phase de finalisation de sa conception architecturale et scénographique. Sa réussite nécessite d’intégrer de multiples facteurs dont le principal est peut-être de trouver un contenu et un langage adaptés au public visé, afin que les enfants de différents âges comme les parents y «trouvent leur compte» et partagent une expérience. En nous appuyant sur le spécimen naturalisé, élément de base du musée d'histoire naturelle, et en l'entourant d'une palette muséographique riche et adaptée, nous souhaitons donner à l'enfant un statut « d'explorateur » lui offrant la possibilité et l'envie de participer activement à la visite pour qu'il vive un moment partagé, fort et enrichissant.

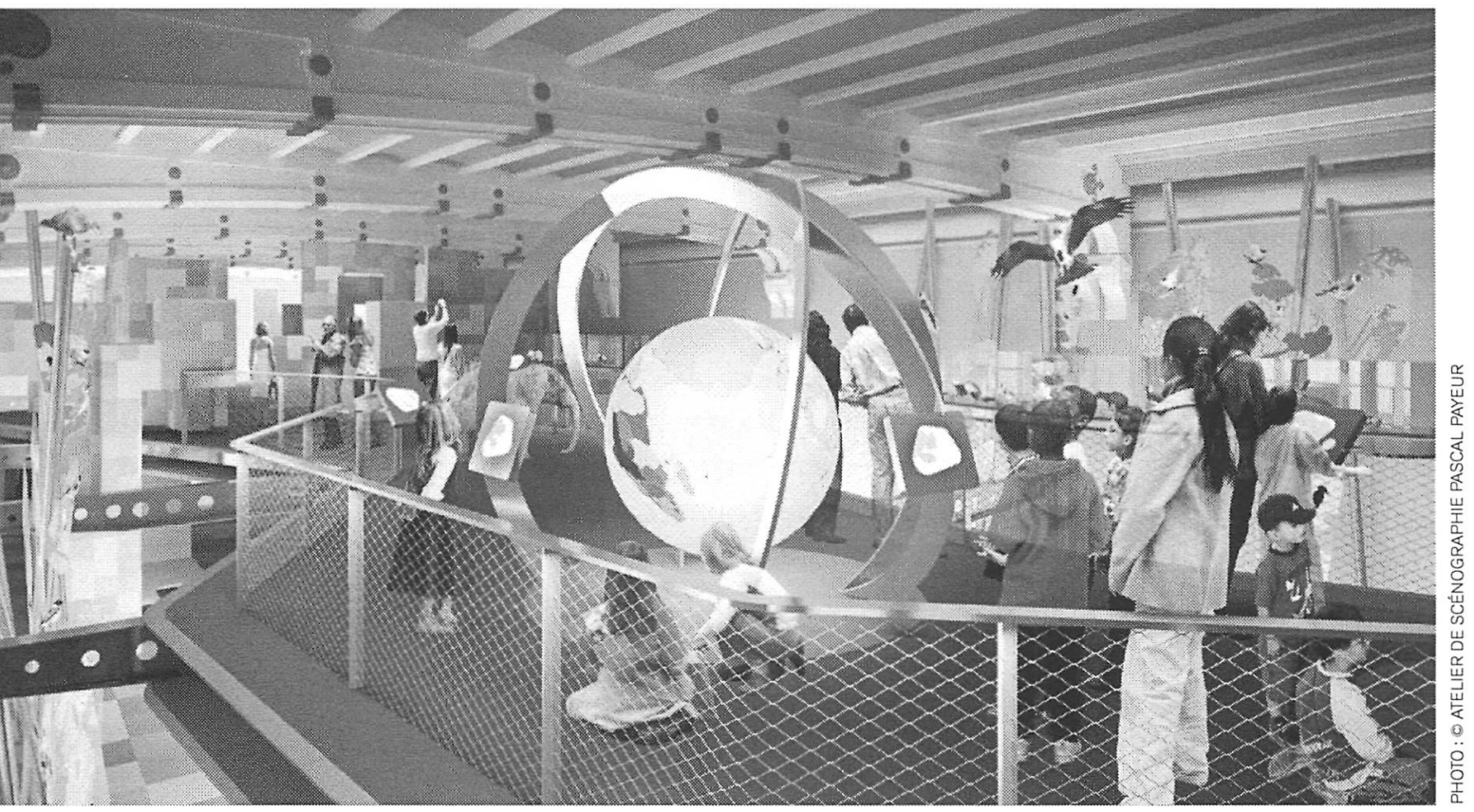

Vue en image de synthèse de la partie forêt tropicale, juillet 08 (image de travail). 
Bibliographie complèmentaire

BOY, Daniel et Anne MUXEL. Les jeunes et la science - Étude sur les attitudes des 11-17 ans à l'égard de la science. Centre d'étude de la vie politique française, Fondation nationale des sciences politiques, Centre national de la recherche scientifique/ CNRS, 1989.

COHEN, Cora. "L'enfant, l'élève, le visiteur, ou la formation au musée". La lettre de l'OCIM (Office de coopération et d'information muséographiques), no 80, 2002, p. 32-37.

EIDELMAN, Jacqueline, Frédérique LAFON et Cécile FROMONT-COLIN.

"Publics en évolution". In : EIDELMAN, Jacqueline et Michel VAN PRAËT (dir.). La muséologie des sciences et ses publics - regards croisés sur la Grande Galerie de l'Évolution du Muséum national d'histoire naturelle. Paris: Presses universitaires de France, 2000, p. 95-121.

GUICHARD, Françoise et Véronique ROUDEAU-LECLERC. "Approches conceptuelles de l'évolution pour les plus jeunes à travers la salle de découverte de la Grande Galerie du Muséum». In : GIORDAN, André, Jean Louis MARTINAND et Daniel RAICHVARG (dir.). Actes des XVIe Journées internationales de l'Éducation scientifique, Chamonix, 1994.

GUICHARD, Jack. "Visiteurs et conception muséographique à la Cité des Enfants". Publics et Musées, no 3, 1993, p. 111-135.

GUICHARD, Jack. "Nécessité d'une recherche éducative dans les expositions à caractère scientifique et technique ". Publics et Musées, no 7, 1995, p. 95-115.

GUICHARD, Jack. "Adapter la muséologie aux enfants". In : SCHIELE, Bernard et Emlyn H. KOSTER (dir.). La révolution de la muséologie des sciences. Lyon: Presses universitaires de Lyon, 1998, p. 207-247.

GUICHARD, Jack et Jean-Louis MARTINAND. Médiatique des sciences. Paris: Presses universitaires de France, 2000.

Ministère de la Culture et de la Communication, Département des études et de la prospective. "Les loisirs des 8-19 ans". Développement culturel, no 131, 1999, 6 p. 


\section{Summary}

[Translated by Allana Carlyle]

Although the number of families visiting the Grande Galerie de l'Évolution is increasing, programming for children remains limited. That is the reason why a project was launched to create an exhibition space dedicated to children.

During the preparatory orientation stage of the project, the authors visited many sites and met museum professionals and early childhood professionals from diverse backgrounds. The authors integrated data from public studies and also considered the technical limitations of the site as well as institutional demands. These different factors lead them to propose a space highlighting the theme of biodiversity designed for intermediate students, ages 6-12, who are either accompanied on a class field trip or on a family visit to the museum.

The exhibition is structured in two large parts, on two different levels. The first level opens directly onto the floor; the second level is up on a towering platform above the first. The visitor is firstly invited to explore three clearly labelled environments or ecosystems, beginning with the city, a familiar environment for most visitors, followed by the river and ending with the tropical forest, an example of a far-off area and a showcase of biodiversity. The topic is next broadened to the global scale, which allows visitors to investigate the Earth's biodiversity, exposes them to global environmental issues, and encourages them to question themselves on the role that each person, individually and collectively, plays in the preservation of the planet. 
The exhibition aims above all to evoke curiosity, to heighten awareness, and to provoke thought. It utilises real objects, in particular animal specimens in their natural environment, to incite emotion and encourage questioning. A partially-guided visit is organized into four successive rooms, the three "ecosystems" and the "planet," within which the visitors can explore freely. It is possible for the exhibition to accommodate a group of 30 , for example a class of students. The visit alternates moments of observation and participation with interactive objects to manipulate either alone or in a group. In the first section, the staging and decor evoke the three environments. In contrasts, the "planet" section up on the platform is largely open and relates both conceptually and physically with the section below.

In brief, the exhibition is of a multi-support style relying on a variety of presentations and devices capable of capturing the interest of a young visitor and promoting interaction amongst children or between children and adults. It makes use of still images and animations, simple and complex mechanical devices, as well as different styles of interactive multimedia animation. 